\title{
Short-Term Pretreatment of Naringin Isolated from Citrus Wilsonii Tanaka Attenuates Rat Myocardial Ischemia/Reperfusion Injury
}

\section{Liping Cheng}

Huazhong Agriculture University

Hangxiu Liu

Yunnan Academy of Agricultural Sciences

\section{Xuefei Li}

Renmin Hospital of Wuhan University: Wuhan University Renmin Hospital

Wenting Liu

Huazhong Agriculture University

\section{Lili Zhao}

Taian City Central Hospital

\section{Hongzhi Du}

Hubei University of Chinese Medicine

Xiaorong Hu

Wuhan University Zhongnan Hospital

Zhaocheng Ma ( $\sim$ mzhaocheng@mail.hzau.edu.cn )

Huazhong Agricultural University: Huazhong Agriculture University

\section{Research Article}

Keywords: Myocardial I/R injury, naringin, anti-inflammation, cardioprotective effect

Posted Date: August 23rd, 2021

DOI: https://doi.org/10.21203/rs.3.rs-817406/v1

License: (c) (1) This work is licensed under a Creative Commons Attribution 4.0 International License. Read Full License 


\section{Abstract}

Pretreatment or treatment with anti-apoptotic, anti-inflammatory, or anti-oxidative approaches could be critical for reducing the occurrence of myocardial I/R injury. Naringin, a natural flavonoid, plays important roles in inflammation-related diseases. Immature dry fruits of Citrus wilsonii Tanaka (Xiang Yuan) are rich in naringin that can be used as traditional Chinese medicine to treat inflammation-related symptoms. However, its roles in cardioprotective role remain unclear. This study aimed to isolate naringin from Citrus wilsonii Tanaka fruit and test their cardioprotective effect. The dry fruits of Citrus wilsonii Tanaka were extracted with boiling water and then supernatants were freeze-dried to yiled aqueous extract (ZQAE). The extract was chemoprofiled using UPLC-MS/MS to stand for major constituents, then subjected to different chromatographic separation steps and naringin was isolated in a high yield. The cardioprotective effect of the aqueous extract of ZQAE and naringin were investigated in a myocardial I/R rat model and to elucidate the mechanism underlying its cardioprotective effect. Naringin was successfully isolated from ZQAE powder by recrystallization in a very high yield. Our results indicated that 5-day ZQAE and naringin pretreatment both promoted histopathological changes and reduced myocardial enzymes induced by I/R. Moreover, the $50 \mathrm{mg} / \mathrm{kg}$ and $100 \mathrm{mg} / \mathrm{kg}$ ZQAE doses pretreatment presented a striking decreased the infarct size as well as myocardial enzyme levels but also inhibited myocardial apoptosis, the inflammatory response and oxidative stress. The cardioprotective effect of $5 \mathrm{mg} / \mathrm{kg}$ dose of naringin pretreatment is comparable with that of $5 \mathrm{mg} / \mathrm{kg}$ drug ditiazem pretreatment. Additionally, Naringin pretreatment exhibited striking decreases in the apoptosis index and downregulation of cleaved-Caspase3 protein expression. Meanwhile, naringin downregulated HMGB1 expression and upregulated SIRT1 expression in the myocardium. These findings suggest that short-term pretreatment with ZQAE and naringin both protect against myocardial I/R injury by suppressing myocardial apoptosis, the inflammatory response and oxidative stress. The cardioprotective effect of naringin involves SIRT1 activation and may interact with HMGB1 and inhibit the release of HMGB1.

\section{Introduction}

Acute myocardial infarction is an epidemic in China and worldwide. Since 2005, its mortality rate has been rapidly increased in China [1]. Currently, emergency reperfusion is the common and most effective therapy for the treatment of acute heart attack $[2,3]$. However, the timely restoration of blood flow after a heart attack could potentially induce a series of adverse reactions, such as cardiocyte apoptosis, inflammatory response, and oxidative stress, leading to aggravation rather than alleviation of myocardial injury [4]. This condition is known as myocardial ischemia/reperfusion (I/R) injury [4]. In recent years, myocardial I/R injury has been a big concern in the treatment of patients with acute myocardial infarction. Pretreatment or treatment with anti-apoptotic, anti-inflammatory, or anti-oxidative approaches could be critical for reducing the occurrence of myocardial I/R injury, which in turn improves the clinical outcome of reperfusion therapy .

Some immature dry fruits of Citrus fruits are used as traditional Chinese medicine to treat inflammationrelated symptoms, such as Citrus grandis 'Tomentosa', Citrus grandis (L.) Osbeck, Poncirus trifoliate, 
Citrus wilsonii Tanaka. It is recorded that the dried fruit of Citrus wilsonii Tanaka fruit is Chinese herbal medicine Fructus Citri. Though the mechanism remains unclear, the powder of dried Citrus wilsonii Tanaka fruit is clinically applied to treat patients with excessive gastric acid secretion, bloating, and digestive health conditions. It has also been used to treat respiratory diseases, such as coughing and phlegmy throat $[5,6]$. Naringin is the most abundant flavonoid in Citrus wilsonii Tanaka fruit with multiple bioactivities. It has been reported to have antioxidant, anti-inflammatory, antiviral and has also exhibited cardioprotective effects in I/R models by reducing oxidative stress and inflammation [7]. In our previous in vitro studies, we reported that Citrus wilsonii Tanaka extracts exhibit a potent anti-inflammatory effect on lipopolysaccharide (LPS)-induced inflammation [8]. Thus, we speculate that Citrus wilsonii Tanaka could have cardioprotective effects on myocardial I/R injury.

Moreover, the evidence on how Citrus wilsonii Tanaka fruit plays its bioactive role in health and disease in vivo is elusive. There is no in vivo data available according to the literature. Myocardial I/R injury in rats is widely used as an in vivo experimental model in acute myocardial infarction researches [9-11]. Herein, we sought to investigate the cardioprotective role of composites from Citrus wilsonii Tanaka fruit in myocardial I/R injury by testing the myocardial infarct size, apoptosis, oxidative stress, inflammatory markers, and regulation of protein expression in myocardial cells in rats.

\section{Materials And Methods}

\subsection{Isolation and extraction compositions from Citrus wilsonii Tanaka fruit}

Citrus wilsonii Tanaka fruits were collected from farms in Nanzheng District of Shaanxi Province with the permission of the owner. The fruit samples were pretreated into powders as previously described [8]. Briefly, ten grams of the powders were macerated and extracted in $1000 \mathrm{~mL}$ distilled water for $1 \mathrm{~h}$ at $95^{\circ} \mathrm{C}$. The extracted supernatants were collected after centrifugation at $3000 \mathrm{~g}$ for $20 \mathrm{~min}$ at $4^{\circ} \mathrm{C}$. Then the yielded pellets were re-extracted twice following the same procedure. Supernatants were then combined, freeze-dried, and resulted in the aqueous extract of Citrus wilsonii Tanaka fruit (ZQAE). Naringin was recrystallized from ZQAE by water and deposited in ethanol and identified by Mass Spectrum. Briefly, the supernatants of the aqueous extract liquid were standing for overnight at $4^{\circ} \mathrm{C}$ and deposited precipitate. The precipitate was collected and preliminary purified by ethanol. The filtrate, on standing at $4^{\circ} \mathrm{C}$, and deposited crystals; the precipitate was collected and re-dissolved with water, on standing, and deposited crystals, repeated the steps for twice. The recrystallized precipitate was obtained and freeze-dried.

\subsection{Analysis of compositions from Citrus wilsonii Tanaka fruit}

ZQAE was profiled as described previously [8]. Briefly, ZQAE was dissolved with distilled water to $1 \mathrm{mg} / \mathrm{mL}$, filtered with a $0.22 \mu \mathrm{M}$ membrane, and subjected to high performance liquid chromatography (HPLC) quantification to measure the contents of naringin, hesperidin, neohesperidin, rhoifolin, naringenin 
and poncirin. The purified naringin identification was carried out by using UPLC-QTOF- MS/MS. The purity was determined using an HPLC method with an external standard.

\subsection{Animals, myocardial ischemia/reperfusion model and experimental design}

All protocols were approved by the Institutional Animal Care and Use Committee of Wuhan University (Approval Number: 2015 - 0563) and performed following the Guideline for the Care and Use of Laboratory Animals published by the US National Institutes of Health (NIH Publication, revised 1996). Male Sprague-Dawley rats weighing 200-250 g were purchased from the Animal Experiment Center of Wuhan University (Wuhan, China).

Myocardial I/R model and experimental design. Rats were anesthetized by $45 \mathrm{mg} / \mathrm{kg}$ body weight (BW) sodium pentobarbital (i.p., Sigma, St. Louis, USA). Then, animals were ventilated artificially with a volume-controlled rodent respirator, and an electrocardiogram was monitored with a computer-based EP system (LEAD2000B, Jinjiang Ltd., Chengdu, China). The myocardial I/R rat model was established as previously described [12]. Rats were euthanized with $22.5 \mathrm{mg} / \mathrm{kg} \mathrm{BW}$ sodium pentobarbital (i.p.), and the blood and heart samples were collected for evaluation of myocardial injury and other biochemical assessments.

Three times animal experiments were carried out. (1) Sprague-Dawley rats were randomly assigned to five groups ( $n=6$ /group) as following: Sham-operated control (Sham): rats were pretreated with $0.9 \%$ $\mathrm{NaCl}$ (solvent for the ZQAE) by intraperitoneal (i.p.) injection for 5 consecutive days before the surgical manipulation without ligation of the left anterior descending coronary artery (LAD). I/R group: rats were treated with $0.9 \% \mathrm{NaCl}$ (i.p.) for 5 consecutive days, and then subjected to LAD occlusion for $30 \mathrm{~min}$ followed by reperfusion for $4 \mathrm{~h}$. Rats were pretreated with ZQAE at 25, 50, or $100 \mathrm{mg} / \mathrm{kg} \mathrm{BW,} \mathrm{by} \mathrm{i.p.}$ injection for 5 consecutive days), and then the LAD was occluded for $30 \mathrm{~min}$ followed by reperfusion for $4 \mathrm{~h}$. (2) Rats were randomly assigned to three groups ( $\mathrm{n}=10 / \mathrm{group}$ ). Doses of ZQAE at $50 \mathrm{mg} / \mathrm{kg}$ were pretreatment for 5 days and then the LAD was occluded for $30 \mathrm{~min}$ followed by reperfusion for $4 \mathrm{~h}$. (3) Rats were randomly assigned to four groups ( $\mathrm{n}=10 / \mathrm{group}$ ). Doses of naringin at $5 \mathrm{mg} / \mathrm{kg}$ and diltiazem at $5 \mathrm{mg} / \mathrm{kg}$ were pretreatment for 5 days and then the LAD was occluded for 30 min followed by reperfusion for $4 \mathrm{~h}$.

\subsection{Histopathological examination}

Myocardial tissues were fixed in 4\% paraformaldehyde (in PBS) and embedded in paraffin. Paraffin sections were stained with hematoxylin and eosin (H\&E). Five fields at 200x magnification were scored in each group to quantify the histological cardiac damage. The scoring rule was described previously [12].

\subsection{Assessment of myocardial injury}

Serum levels of creatine kinase-MB (CK-MB) and cardiac troponin I (cTnl) were measured to assess the myocardial injury. Standard techniques were performed using commercial kits for CK-MB and cTnl 
(Nanjing Jiancheng Bioengineering Institute, Nanjing, China).

\subsection{Assessment of infarct size}

The infarct size was measured using the 2,3,5-triphenyl tetrazolium chloride staining (TTC, Sigma-Aldrich, St. Louis, USA) as previously described [11]. Briefly, after reperfusion, LAD has occluded again, and the rats were injected with $2 \mathrm{ml}$ of $1 \%$ Evans blue dye (Sigma-Aldrich, St. Louis, USA) via the femoral vein. The hearts were removed from the body, frozen at $-80^{\circ} \mathrm{C}$ for $15 \mathrm{~min}$ and then cut (approximately $2 \mathrm{~mm}$ ) into five slices from the apex to the base. The slices were immersed and incubated in 1\% TTC for 20 min at $37^{\circ} \mathrm{C}$ to separate the infarct area (white) from the risk area (red) and then fixed in $4 \%$ paraformaldehyde. The white tissues and red tissues from each section were determined with image analysis software (Image-Pro Plus 6.0, Media Cybernetics, Silver Spring, USA). Five rats per group were used to measure the myocardial infarct size. The infarct size (\%) was expressed as: infarct area / (risk+ infarct) area $\times 100 \%$.

\subsection{TUNEL assay}

Myocardial apoptosis was analyzed by the TUNEL assay using a commercial kit (Roche Applied Science, Indianapolis, USA) according to the manufacturer's instructions. The apoptosis index was defined by the percentage of apoptotic cell number/total cell number, as calculated using Image-Pro Plus 6.0 software. Notably, brown staining in the nucleus indicated apoptosis.

\subsection{Measurement of oxidative stress and inflammatory cytokines in myocardial tissues}

Oxidative stress markers, including malondialdehyde (MDA) contents and antioxidant superoxide dismutase (SOD) activities, were determined spectrophotometrically in myocardial tissues, using commercial kits (Nanjing Jiancheng Bioengineering Institute, Nanjing, China) according to the manufacturer's instructions. Production of tumor necrosis factor-a (TNF-a) and interleukin-6 (IL-6) was assessed in ischemic areas of the left ventricle by enzyme-linked immunosorbent assays according to manufacturer's instructions (Nanjing Jiancheng Bioengineering Institute, Nanjing, China).

\subsection{Western blot}

Frozen ischemic areas of left ventricle samples were analyzed by Western blot analysis. The antibody against interleukin-23 (IL-23) (1:800) was purchased from Proteintech Group Inc, USA. Anti-totalCaspase3 (1:1000) was purchased from Abcam (UK). Anti-cleaved-Caspase3 (1:1000), anti-p38 (1:1000), and anti-p-p38 (1:1000) were obtained from Cell Signaling Technology, Danvers, USA. Anti-high mobility group box 1 (HMGB1) (1:300) and anti-glyceraldehyde-3-phosphate dehydrogenase (GAPDH, 1:1000) were received from the Boster, Wuhan, China.

\subsection{Statistical analysis}

SPSS 19.0 was used for data analysis. All data are expressed as mean \pm SD. Student's t-test was used for comparison between two groups. Multiple comparisons among groups were evaluated by one-way 
ANOVA or a Welch test. Student-Newman-Keuls or Dunnett's T3 test was used for post hoc multiple comparisons. $P$ value $<0.05$ was considered statistically significant.

\section{Results}

\subsection{Identification of composites form ZQAE and isolation of naringin}

Chinese herbal medicines are usually boiled into decoctions. The dry fruits of Citrus wilsonii Tanaka were extracted with boiling water and then supernatants were freeze-dried to yiled aqueous extract (ZQAE). HPLC was performed to profile the flavonoids in ZQAE. As shown in Fig. 1A, six flavonoids naringin, hespiridin, neohesperidin, rhoifolin, naringenin, poncirin were measured. Naringin is one of the most important naturally-occurring flavonoids and it was the most predominant found in ZQAE flavonoids. The aqueous extract (ZQAE) was chemoprofiled using UPLC-MS/MS to stand for major constituents, then subjected to different chromatographic separation steps. Naringin was isolated from ZQAE powder by recrystallization. The precipitate was collected and re-dissolved with water, and deposited crystals. The structure of naringin was verified by MS spectrum and the purity was determined to be $>99 \%$ using an HPLC method with an external standard (Fig. 1B). Naringin was isolated in a very high yield from Citrus wilsonii Tanaka fruit representing a good source for commercial level.

\subsection{ZQAE pretreatment promoted histopathological changes and attenuated myocardial injury}

After 5 days of ZQAE pretreatment, histopathological changes were detected by using H\&E staining, as shown in Fig. 2. Compared with the I/R group, less myocardial fiber disruption, necrosis, and inflammatory cell infiltration were observed in the ZQAE groups (Fig. 2A). The myocardial damage in the ZQAE groups was less than that in the I/R group. To further assess the myocardial injury, the damage score and levels of CK-MB and CTnl in serum samples were determined.Compared to the Sham group, myocardial I/R induced a markedly increased damage score (Fig. 2B) and caused an increase in levels of serum CK-MB and CTnl (Fig. 2C and D). ZAQE (25, 50, and $100 \mathrm{mg} / \mathrm{kg} \mathrm{BW}$ ) pretreatments at different doses exhibited significant protective effects on lowered damage scores and decreased serum CK-MB and cTnl levels (Fig. $2 \mathrm{C}$ and D). The results suggested that ZQAE conferred a favorable role in attenuating myocardial I/R injury. Additionally, among the three different doses of ZQAE pretreatments, both ZQAE50I/R and ZQAE100-I/R groups presented a striking difference compared to the ZQAE25-I/R group. However, no significant difference was observed between these two groups. Hence, the dose of $50 \mathrm{mg} / \mathrm{kg} \mathrm{BW}$ ZQAE was chosen for the following experiments.

\subsection{ZQAE pretreatment reduced myocardial I/R injury, ameliorated myocardial oxidative stress and inhibited}




\section{inflammatory responses}

The cardioprotective effects of ZQAE (50 mg/kg BW) against myocardial I/R injury were investigated. TTC staining results showed that the myocardial infarction size was significantly smaller in the ZQAE50I/R group compared with the I/R group (data not shown). As shown in Fig. 3, myocardial I/R significantly increased the MDA content and decreased the activities of SOD, indicating oxidative stress occurred in the hearts of myocardial I/R injury rats. Compared with the I/R group, ZQAE pretreatment markedly decreased the MDA content and increased the anti-oxidative capacity by upregulating SOD activities, suggesting the anti-oxidative effect of ZQAE.

The effect of ZQAE pretreatment on the inflammatory responses after myocardial I/R was shown in Fig. 3. Compared with the Sham group, myocardial I/R increased the IL-23 expression and levels of TNF- $a$ and IL-6. ZQAE significantly decreased levels of IL- 6 and TNF- $a$ and suppressed IL-23 expression caused by myocardial I/R, compared to that of the I/R group without ZQAE, suggesting that ZQAE pretreatment suppressed inflammatory response during myocardial I/R. These results indicated that the ZQAE pretreatment could reduce myocardial I/R injury.

\subsection{Naringin pretreatment reduced myocardial I/R injury, ameliorated myocardial oxidative stress and inhibited inflammatory responses}

The cardioprotective effect of naringin was investigated and to elucidate the mechanism underlying its cardioprotective effect. The myocardial infarction size was significantly smaller in the naringin-l/R (dose $5 \mathrm{mg} / \mathrm{kg}$ ) and ditiazem-1/R (dose $5 \mathrm{mg} / \mathrm{kg}$ ) groups compared with the I/R group (Fig. 4A). Additionally, myocardial I/R injury increased concentrations of serum CK-MB and cTnl. Intriguingly, the naringin and ditiazem pretreatment significantly reversed these changes caused by myocardial I/R (Fig. 4B and 4C). These results indicated that the naringin pretreatment could reduce myocardial $I / R$ injury .

As shown in Figs. 5, naringin pretreatment markedly decreased the MDA content and increased the antioxidative capacity by upregulating SOD activities, suggesting the anti-oxidative effect of naringin. Naringin significantly decreased levels of IL- 6 and TNF- $a$ caused by myocardial I/R, suggesting that naringin pretreatment suppressed inflammatory responses during myocardial I/R(Fig. 5A and 5C). These results indicated that the naringin pretreatment could reduce myocardial I/R injury and be comparable with drug ditizem.

\subsection{Naringin pretreatment suppressed myocardial apoptosis}

The myocardial apoptosis by assessing the apoptosis-related proteins using TUNEL assays and expression of cleaved-caspase 3 by western blot were measured (Fig. 6). Compared with the Sham group, myocardial I/R markedly increased the apoptosis index and cleaved-Caspase3 protein expressions. 
Naringin pretreatment exhibited striking decreases in the apoptosis index and downregulation of cleavedCaspase3 protein expression compared with those of the I/R group. It indicated that naringin could reduce myocardial apoptosis.

\subsection{Naringin pretreatment activated the SIRT1 pathway and suppressed HMGB1 exppression}

Finally, the possible underlying mechanisms by which naringin protected against myocardial I/R injury were investigated. As shown in Fig. 7, 1/R caused a significant increase in protein expression levels of HMGB1, compared to the Sham group. Naringin significantly reversed the l/R effects on the expression of HMGB1 proteins. Meanwhile, naringin significantly increased SIRT1 expression after suppressed by I/R, indicating that naringin could activate the SIRT1 pathway and inhibit HMGB1 expression.

\section{Discussion}

In recent years, more attention is attracted to natural active ingredients from plants, especially herbal medicines, which should be the important resources for the treatment of myocardial I/R injury [13-16]. For instance, it was found that the saffron aqueous extract prevented myocardial I/R injury by regulating Nrf2 activity in mice [13]. The aqueous extract isolated from Theobroma grandiflora seeds mitigates myocardial I/R injury by controlling eNOS activation [14]. We found that aqueous extract from Citrus wilsonii Tanaka fruit protected myocardium against I/R injury. It is noteworthy that a low dose ZQAE pretreatment for a short time exhibited a marked improvement of the myocardial function in rat models, compared with other relevant researches $[13,17]$. Such as the saffron aqueous extract pretreated mice at a dose of $60 \mathrm{mg} / \mathrm{kg}$ for 4 weeks [13], intragastric administration of $600 \mathrm{mg} / \mathrm{kg}$ rosa rugosa flavonoids for one week [17]. In the current study, the 5-day pretreatment of $\operatorname{ZQAE}(25,50$, and $100 \mathrm{mg} / \mathrm{kg} \mathrm{BW} /$ day $)$ significantly improved the pathomorphological changes in myocardium tissue and mitigated myocardial injury induced by $\mathrm{I} / \mathrm{R}$. The results suggested that a short-term ZQAE preconditioning could be sufficient to protect rats from myocardial I/R injury. These intriguing findings may indicate a high potential of Citrus wilsonii Tanaka fruit in the treatment of myocardial I/R injury.

Flavonoids are a group of common phytochemicals with anti-oxidative, anti-inflammation, and antiapoptosis properties $[8,18-20]$. In the present study, flavonoids were the most dominant metabolites in ZQAE, and among them, naringin is the most abundant. These results are consistent with our previous report [8]. Numerous publications have presented that flavonoids have beneficial effects on myocardial protection and could prevent myocardial I/R injury [16, 21-24]. The previous studies have demonstrated that citrus-derived flavonoids have beneficial effects on myocardial protection [12, 25, 26]. Our previous work indicated that hesperidin could protect the myocardium in I/R injury via regulating the PI3K/Akt pathway [12]. Naringin has been reported to protect the myocardium from I/R injury through suppression of inflammation and regulation of Hsp27, Hsp70, p-eNOS/p-Akt/p-ERK signaling [25]. HMGB1 played a critical role in inflammatory responses [27-29] and participated in the cardiomyocyte apoptosis corresponding with toll-like receptor 4 during myocardial I/R injury [30]. Down-regulation of HMGB1 
expression potentially attributed to attenuation of myocardial I/R injury [12, 31, 32]. HMGB1 expression could be inhibited by flavonoids, such as hesperidin [11], nobiletin [33], quercetin [34, 35], and kaempferol [36]. In the present study, we found that naringin pretreatment markedly inhibited myocardial I/R injuryenhanced HMGB1 protein expression, as well as cardiomyocyte apoptosis and inflammatory responses. These results imply that naringin may alleviate myocardial I/R injury by inhibiting HMGB1 activation in rats. SIRT1 is a type of histone deacetylase, which is specifically involved in its protective effect against myocardial I/R injury. SIRT1 regulated the FOXO1 to affect apoptotic pathways in the heart. Hsu [37]confirmed that SIRT1 protects against myocardial I/R injury by upregulating antioxidants and decreasing oxidative stress. Our data showed that naringin significantly reduced the myocardial infarct size, inhibited cardiomyocyte apoptosis, suppressed inflammatory responses, attenuated oxidative stress, and upregulation of SIRT1. Interestingly, SIRT1 directly interacted with HMGB1 via its N-terminal lysine residues, and thereby inhibited HMGB1 release to improve survival in an experimental model of sepsis [38]. In our study, we found that SIRT1 expression is significantly upregulated after naringin pretreatment, accompanied by downregulated HMGB1 expression. All these results suggested that the cardioprotection of Citrus wilsonii Tanaka fruit and naringin treatment involves SIRT1 activation and naringin-induced SIRT1 activation may interact with HMGB1 and inhibit the release of HMGB1.

\section{Conclusion}

In conclusion, naringin was successfully isolated from Citrus wilsonii Tanaka fruit by recrystallization in a very high yield. 5-day ZQAE and naringin pretreatment both promoted histopathological changes and reduced myocardial enzymes induced by I/R. Moreover, the $50 \mathrm{mg} / \mathrm{kg}$ and $100 \mathrm{mg} / \mathrm{kg}$ ZQAE doses pretreatment presented a striking decreased the infarct size as well as myocardial enzyme levels but also inhibited myocardial apoptosis, the inflammatory response and oxidative stress. The cardioprotective effect of $5 \mathrm{mg} / \mathrm{kg}$ dose of naringin pretreatment is comparable with that of $5 \mathrm{mg} / \mathrm{kg}$ drug ditiazem pretreatment. Additionally, Naringin pretreatment exhibited striking decreases in the apoptosis index and downregulation of cleaved-Caspase3 protein expression. Meanwhile, naringin downregulated HMGB1 expression and upregulated SIRT1 expression in the myocardium. These findings suggest that short-term pretreatment with ZQAE and naringin both protect against myocardial I/R injury by suppressing myocardial apoptosis, the inflammatory response and oxidative stress. The cardioprotective effect of naringin involves SIRT1 activation and may interact with HMGB1 and inhibit the release of HMGB1.

\section{Declarations}

\section{Ethics approval and consent to participate}

The animal experimental design and protocols used in this study were approved by the Institutional Animal Care and Use Committee of Wuhan University (Approval Number: 2015-0563) and performed following the Guideline for the Care and Use of Laboratory Animals published by the US National Institutes of Health (NIH Publication, revised 1996). 


\section{Consent for publication}

Not applicable.

\section{Competing interests}

The authors declare that they have no competing interests.

\section{Acknowledgements}

The study was supported by the National Key Research and Development Program (2018YFD1000200; 2019YFD1001400), the ability establishment of sustainable use for valuable Chinese medicine resources (2060302), and Fundamental Research Funds for the Central Universities (2662020YLPY007).

\section{Authors' contributions}

The authors declare that all data were generated in-house and that no paper mill was used. HXR and MZC conceived and designed the experiments; CLP, LXF and LWT performed the experiments; CLP and LHX wrote the paper; CLP, LXF, ZLL, HXR analyzed the data and discussed the results. All authors read and approved the final manuscript.

\section{References}

1. Chen, W.W.; Gao, R.L.; Liu, L.S.; Zhu, M.L.; Wang, W.; Wang,Y.J.; Wu, Z.S.; Li, H.J.; Gu, D.F.; Yang, Y.J. China cardiovascular diseases report 2015: a summary. J Geriatr Cardiol. 2017,14(1):1-10.

2. Hausenloy, D.J.; Yellon, D.M. Myocardial ischemia-reperfusion injury: a neglected therapeutic target. $J$ Clin Invest. 2013, 123(1):92-100.

3. Kloner, R.A.; Shi, J.; Dai, W.;Carreno, J.; Zhao, L. Remote ischemic conditioning in acute myocardial infarction and shock states. J Cardiovasc Pharmacol Ther. 2019, 1074248419892603.

4. Yellon, D.M.; Hausenloy, D.J. Myocardial reperfusion injury. New Engl J Med. 2007, 357(11):11211135.

5. Chinese Pharmacopoeia Commission Pharmacopeia of the People's Republic of China. Chemical Industry Press. 2010, 242.

6. Dan, B.; Andrew, G. Chinese Herbal Medicine, 8th edn. Eastland Press. 1986, 334-335.

7. Neha, Rani. Regulation of heat shock proteins 27 and 70, p-Akt/p-eNOS and MAPKs by Naringin Dampens myocardial injury and dysfunction in vivo after ischemia/reperfusion. PLOS ONE. 2017, $8(12)$.

8. Cheng, L.P.; Ren, Y.J.; Lin D.B.; Peng, S.A.; Zhong, B., Ma, Z.C. The anti-inflammatory properties of Citrus wilsonii Tanaka extract in LPS-induced RAW 264.7 and primary mouse bone marrow-derived dendritic cells. Molecules. 2017, 22(7). 
9. Ibarrola, J.; Matilla, L.; Martinez-Martinez, E.; Gueret ,A.; Fernandez-Celis, A.; Henry, J.P.; Nicol, L.; Jaisser, F.; Mulder, P.; Ouvrard-Pascaud A. Myocardial injury after ischemia/reperfusion is attenuated by pharmacological Galectin-3 inhibition. Sci Rep. 2019, 9(1):9607.

10. Wu, Y.; Leng, Y.; Meng, Q.; Xue, R.; Zhao, B.; Zhan, L.; Xia, Z. Suppression of excessive histone deacetylases activity in diabetic hearts attenuates myocardial ischemia/reperfusion injury via mitochondria apoptosis pathway. J Diabetes Res. 2017, 2017:8208065.

11. Cerkezkayabekir, A.; Sanal, F.; Bakar, E.; Ulucam, E.; Inan, M. Naringin protects viscera from ischemia/reperfusion injury by regulating the nitric oxide level in a rat model. Biotech Histochem. 2017, 92(4):252-263.

12. Li, X.; Hu, X.; Wang, J.; Xu, W.; Yi, C.; Ma, R.; Jiang, H. Short-term hesperidin pretreatment attenuates rat myocardial ischemia/reperfusion injury by inhibiting high mobility group box 1 protein expression via the PI3K/Akt pathway. Cell Physiol Biochem. 2016, 39(5):1850-1862.

13. Efentakis, P.; Rizakou, A.; Christodoulou, E.; Chatzianastasiou, A.; Lopez, M.G.; Leon, R.; Balafas, E.; Kadoglou, N.P.E.; Tseti, I.; Skaltsa, H. Saffron (Crocus sativus) intake provides nutritional preconditioning against myocardial ischemiaereperfusion injury in Wild Type and $\mathrm{ApoE}((-/-))$ mice: Involvement of Nrf2 activation. Nutr Metab Cardiovasc Dis. 2017, 27(10):919-929.

14. Fantinelli, J.C.; Cuéllar, Álvarez, L.N.; González, Arbeláez, L.F.; Ciocci, Pardo, A.; Galeano, García, P.L.; Schinella, G.R.; Mosca, S.M. Acute treatment with copoazú fermented extract ameliorates myocardial ischemia-reperfusion injury via eNOS activation. J Funct Foods. 2017, 34:470-477.

15. Guo, D.; Murdoch, C.E.; Liu, T.; Qu, J.; Jiao, S.; Wang, Y.; Wang, W.; Chen, X. Therapeutic angiogenesis of Chinese herbal medicines in ischemic heart disease: A Review. Front Pharmacol. 2018, 9:428.

16. Xiao, G.; Lyu, M.; Wang, Y.; He, S.; Liu, X.; Ni, J.; Li, L.; Fan, G.; Han, J.; Gao, X. Ginkgo flavonol glycosides or ginkgolides tend to differentially protect myocardial or cerebral ischemia-reperfusion injury via regulation of TWEAK-Fn14 signaling in heart and brain. Front Pharmacol. 2019, 10:735.

17. Zhang, X.; Wang, Y.; Shen, W.; Ma, S.; Chen, W.; Qi, R. Rosa rugosa flavonoids alleviate myocardial ischemia reperfusion injury in mice by suppressing JNK and p38 MAPK. Microcirculation. 2017, 24(7):e12385.

18. Bondonno, N.P.; Bondonno, C.P.; Blekkenhorst, L.C.; Considine, M.J.; Maghzal, G.; Stocker, R.; Woodman, R.J.; Ward, N.C.; Hodgson, J.M.; Croft, K.D. Flavonoid-rich apple improves endothelial function in individuals at risk for cardiovascular disease: A randomized controlled clinical trial. Mol Nutr Food Res. 2018, 62(3):1700674.

19. He, Y.; Xia, Z.; Yu, D.; Wang, J.; Jin, L.; Huang, D.; Ye, X.; Li, X.; Zhang, B. (2019) Hepatoprotective effects and structure-activity relationship of five flavonoids against lipopolysaccharide/Dgalactosamine induced acute liver failure in mice. Int Immunopharmacol. 2019, 68:171-178.

20. Tsai, M.S.; Wang, Y.H.; Lai, Y.Y.; Tsou, H.K.; Liou, G.G.; Ko, J.L.; Wang, S.H. (2018) Kaempferol protects against propacetamol-induced acute liver injury through CYP2E1 inactivation, UGT1A1 activation, and attenuation of oxidative stress, inflammation and apoptosis in mice. Toxicol Lett. 2018, 290:97109. 
21. Li, Q.; Tursun, D.; Shi, C.; Heyrulla, M.; Zhang, X.; Yang, W. Ziziphora clinopodioides flavonoids protect myocardial cell damage from myocardial ischemia-reperfusion injury. Evid Based Complement Alternat Med. 2018, 1-9.

22. Pongkan, W.; Takatori, O.; Ni, Y.; Xu, L.; Nagata, N.; Chattipakorn, S.C.; Usui, S.; Kaneko, S.; Takamura, M.; Sugiura, M. beta-Cryptoxanthin exerts greater cardioprotective effects on cardiac ischemiareperfusion injury than astaxanthin by attenuating mitochondrial dysfunction in mice. Mol Nutr Food Res. 2017, 61(10).

23. Zeng, C.; Jiang, W.; Yang, X.; He, C.; Wang, W.; Xing, J. Pretreatment with total flavonoide extract from Dracocephalum Moldavica L. attenuates ischemia reperfusion-induced apoptosis. Sci Rep. 2018, 8(1):17491.

24. Zhang, H.J.; Chen, R.C.; Sun, G.B.; Yang, L.P.; Zhu, Y.D.; Xu, X.D.; Sun, X.B. Protective effects of total flavonoids from Clinopodium chinense (Benth.) 0 . Ktze on myocardial injury in vivo and in vitro via regulation of Akt/Nrf2/HO-1 pathway. Phytomedicine. 2018, 40:88-97.

25. Rani, N,; Bharti, S.; Manchanda, M.; Nag, T.C.; Ray, R.; Chauhan, S.S.; Kumari, S.; Arya, D.S. Regulation of heat shock proteins 27 and 70, p-Akt/p-eNOS and MAPKs by Naringin Dampens myocardial injury and dysfunction in vivo after ischemia/reperfusion. PLoS One. 2013, 8(12):e82577.

26. Zhou, Z.; Zhang, Y.; Lin, L.; Zhou, J. (2018) Apigenin suppresses the apoptosis of H9C2 rat cardiomyocytes subjected to myocardial ischemiareperfusion injury via upregulation of the PI3K/Akt pathway. Mol Med Rep. 2018, 18(2):1560-1570.

27. Andrassy, M., Volz, H.C.; Igwe, J.C.; Funke, B.; Eichberger, S.N.; Kaya, Z.; Buss, S.; Autschbach, F.; Pleger, S.T.; Lukic, I.K. High-mobility group box-1 in ischemia-reperfusion injury of the heart. Circulation. 2008, 117(25):3216.

28. Sun, N.; Wang, H.; Wang, L. Protective effects of ghrelin against oxidative stress, inducible nitric oxide synthase and inflammation in a mouse model of myocardial ischemia/reperfusion injury via the HMGB1 and TLR4/NF-KB pathway. Mol Med Rep. 2016, 14(3):2764-2770.

29. Yang, Y.F.; Peng, K.; Liu, H.; Meng, X.W.; Zhang, J.J.; Ji, F.H. Dexmedetomidine preconditioning for myocardial protection in ischaemia-reperfusion injury in rats by downregulation of the high mobility group box 1-toll-like receptor 4-nuclear factor kappa B signalling pathway. Clin Exp Pharmacol Physiol. 2017, 44(3):353-361.

30. Ding, H.S.; Yang, J.; Chen, P.; Yang, J.; Bo, S.Q.; Ding, J.W.; Yu, Q.Q. The HMGB1-TLR4 axis contributes to myocardial ischemia/reperfusion injury via regulation of cardiomyocyte apoptosis. Gene. 2013, 527(1):389-393.

31. Hu, X.; Cui, B.; Zhou, X.; Xu, C.; Lu, Z.; Jiang, H. Ethyl pyruvate reduces myocardial ischemia and reperfusion injury by inhibiting high mobility group box 1 protein in rats. Mol Biol Rep. 2012, 39(1):227-231.

32. Hu, X.; Ma, R.; Lu, J.; Zhang, K.; Xu, W.; Jiang, H.; Da, Y. IL-23 Promotes myocardial I/R injury by increasing the inflammatory responses and oxidative stress reactions. Cell Physiol Biochem. 2016, 38(6):2163-2172. 
33. Li, W.; Wang, X.; Niu, X.; Zhang, H.; He, Z.; Wang, Y.; Zhi, W.; Liu, F. Protective effects of nobiletin against endotoxic shock in mice through inhibiting TNF-a, IL-6, and HMGB1 and regulating NF-KB pathway. Inflammation. 2016, 39(2):786-797.

34. Li, X.; Liu, H.C.; Yao, Q.Y.; Xu, B.L.; Zhang, S.C.; Tu, C.T. Quercetin protects mice from ConA-induced hepatitis by inhibiting HMGB1-TLR expression and down-regulating the nuclear factor kappa B pathway. Inflammation. 2016, 39(1):96-106.

35. Cui, W.J.; Hu, G.; Peng, J.; Mu, L.; Liu, J.; Qiao, L.J. Quercetin exerted protective effects in a rat model of sepsis via inhibition of reactive oxygen species (ROS) and downregulation of high mobility group box 1 (HMGB1) protein expression. Med Sci Monit. 2019, 25:5795-5800.

36. Cheng, X.; Yang, Y.L.; Yang, H.; Wang, Y.H.; Du, G.H. (2018) Kaempferol alleviates LPS-induced neuroinflammation and BBB dysfunction in mice via inhibiting HMGB1 release and down-regulating TLR4/MyD88 pathway. Int Immunopharmacol. 2018, 56:29-35.

37. Hsu, C.P.; Zhai, P.; Yamamoto, T. Silent information regu-lator 1 protects the heart from ischemia/reperfusion. Circula-tion. 2010, 122:2170-2182.

38. Hwang, J.S. Deacetylation-mediated interaction of SIRT1-HMGB1 improves survival in a mouse model of endotoxemia. Scientific reports. 2015, 5 : 15971.

\section{Figures}



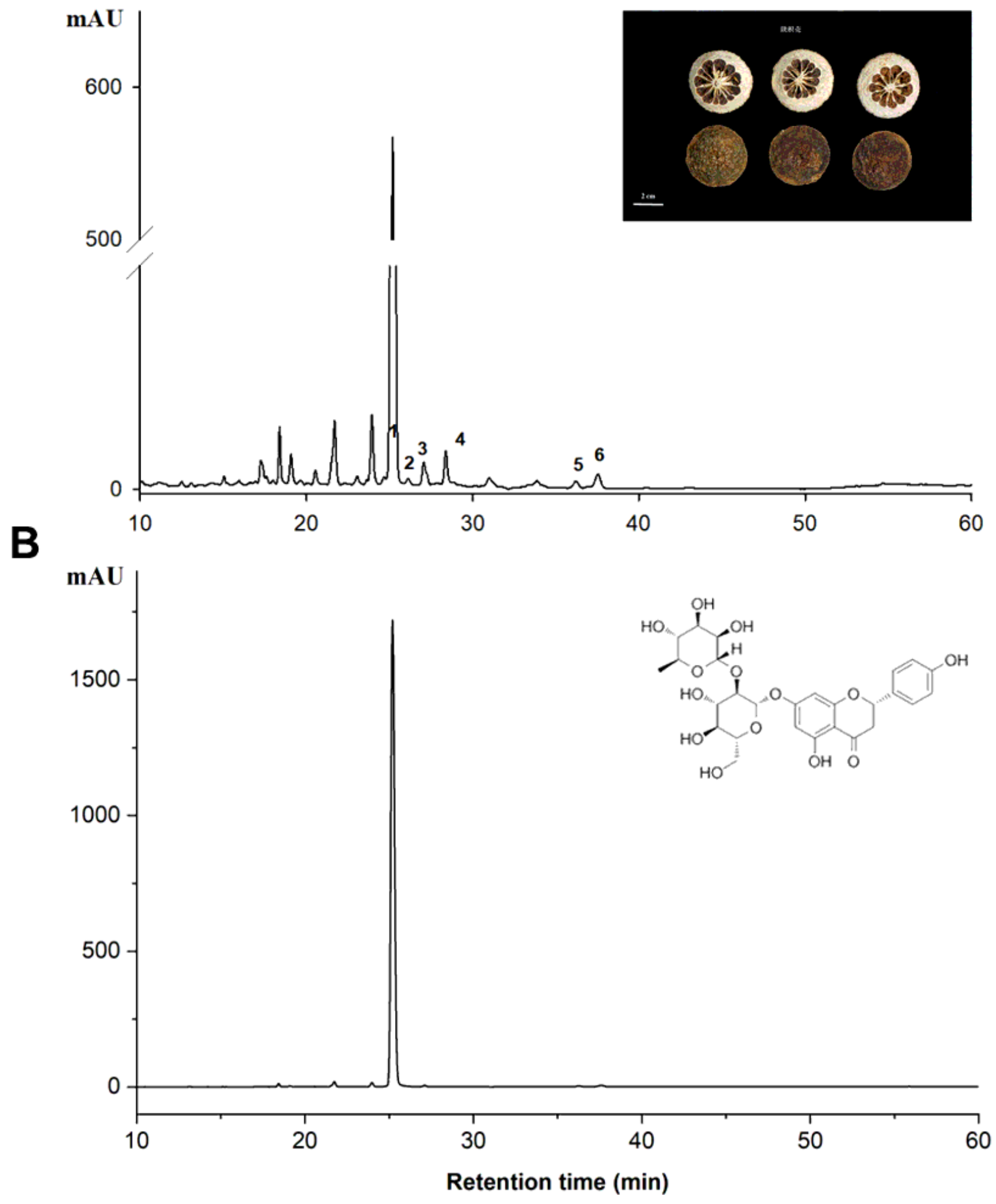

Figure 1

A. High performance liquid chromatography analysis results of Flavonoids in aqueous extract from Citrus wilsonii Tanaka fruit at $283 \mathrm{~nm}$. 1: Naringin, 2: Hespiridin, 3: Neohesperidin, 4: Rhoifolin, 5: Naringenin, 6: Poncirin. B. High performance liquid chromatography analysis results of purified naringin at $283 \mathrm{~nm}$. C. Representative images of Citrus wilsonii Tanaka in southern Shaanxi. 
A
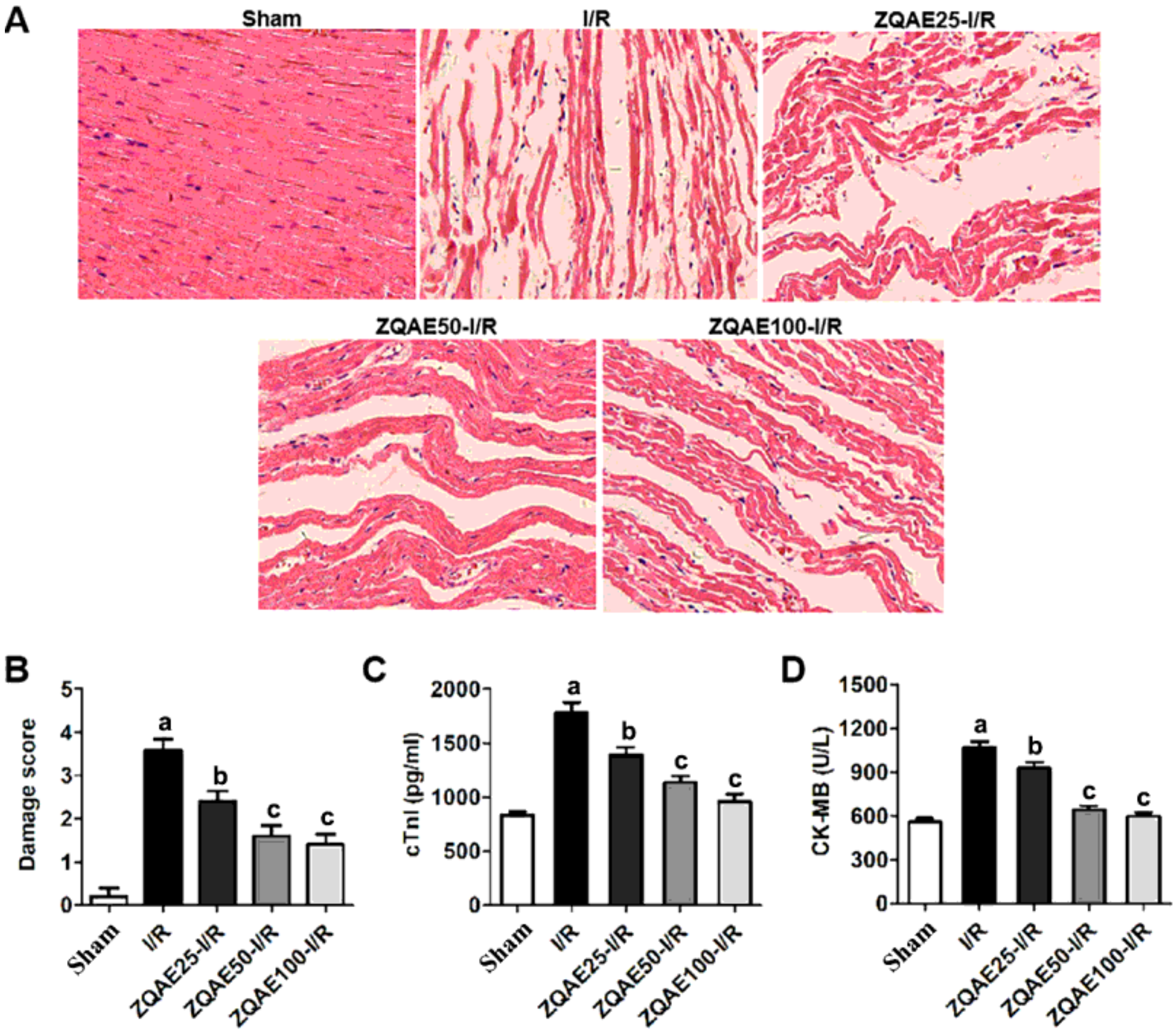

Figure 2

ZQAE pretreatment promotes histopathological changes and reduces myocardial injury after I/R. (A) Representative images of H\&E stained samples $(\times 200)$ demonstrating histopathological changes in the myocardium; the orange arrows indicate ruptured cardiac muscle fibers, and the black arrows indicate hemorrhage. (B) Damage score $(n=5)$. (C) Serum cTnl level $(n=6)$. (D) Serum CK-MB level $(n=6)$. a $p<0.05$ vs. the Sham group; $b p<0.05$ vs. the I/R group; $c p<0.05$ vs. the ZQAE25-I/R group. CK-MB, creatine kinase-MB; CTnl, cardiac troponin l; H\&E, hematoxylin and eosin; I/R, ischemia/reperfusion; Sham, Shamoperated control; ZQAE, aqueous extract of Citrus wilsonii Tanaka fruit . 
A

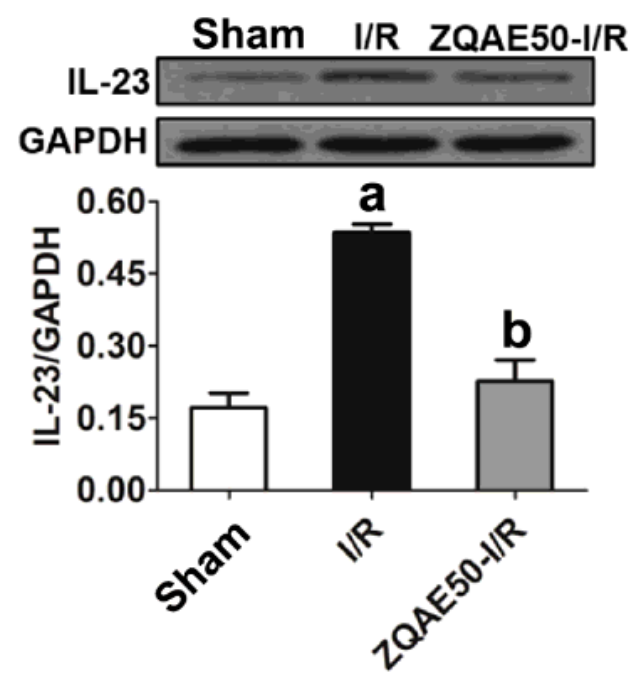

D

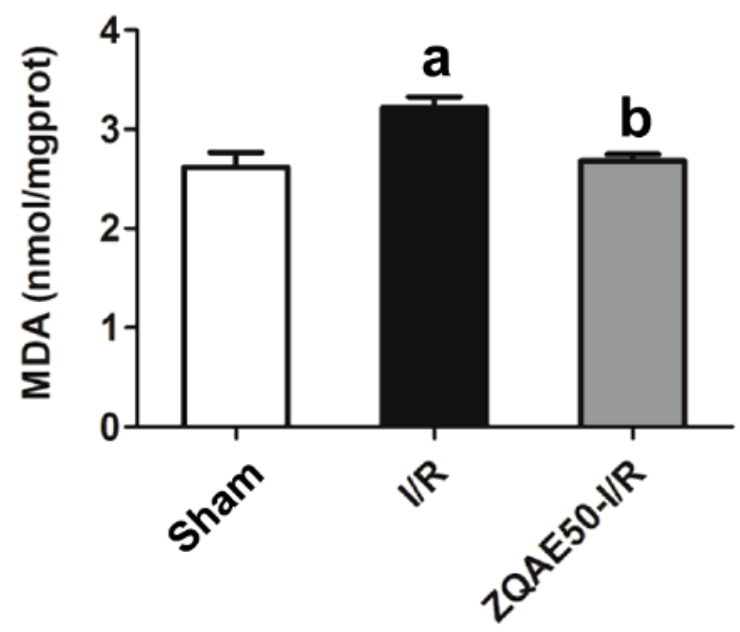

C
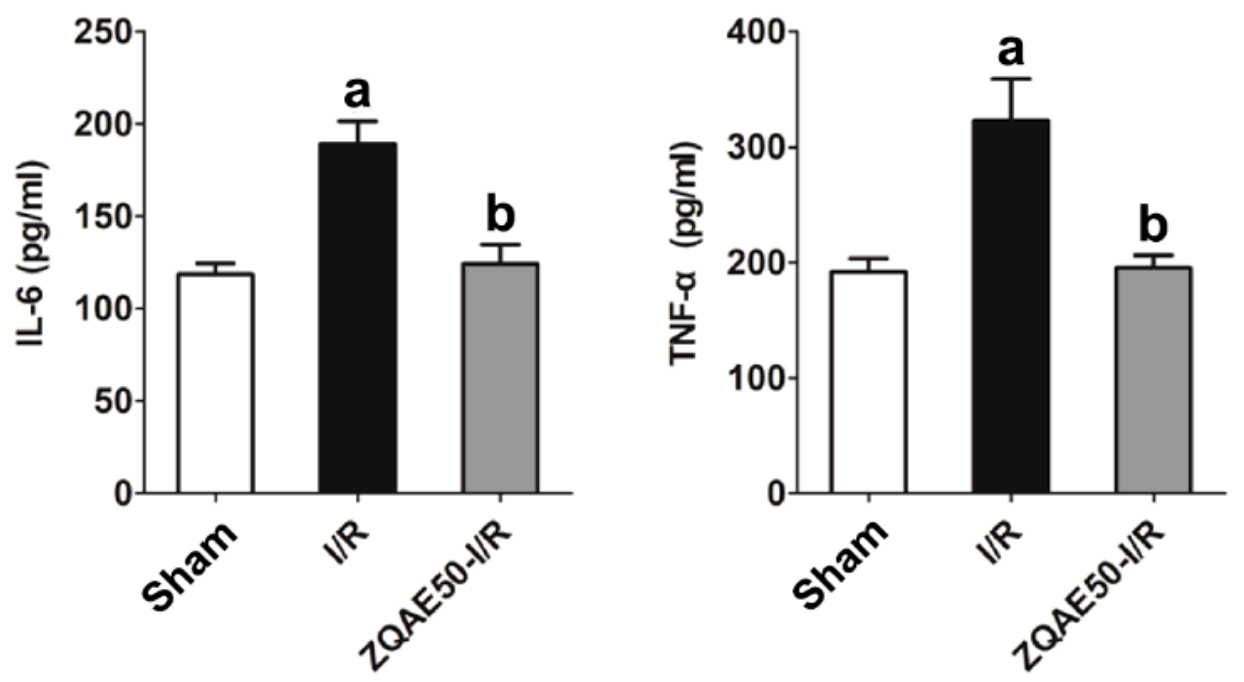

$\mathbf{E}$

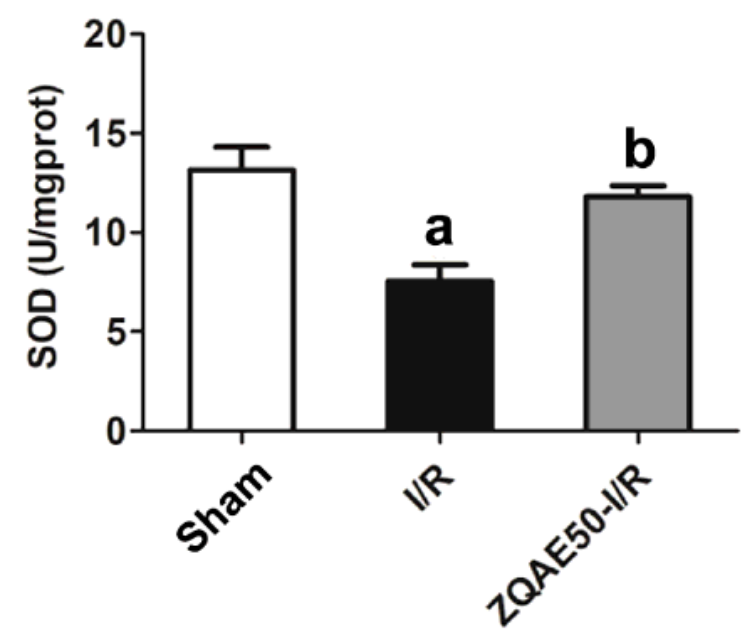

Figure 3

ZQAE pretreatment inhibits the inflammatory response induced by $\mathrm{I} / \mathrm{R}$ and pretreatment ameliorates oxidative stress after myocardial I/R injury. (A) The expression levels of IL-23 $(n=5)$. (B) Level of IL-6 $(n=5)$. (C) Level of TNF-a (n=5). (D) MDA content $(n=5)$. (E) SOD activity $(n=5)$. a $p<0.05$ vs. the Sham group; bp < 0.05 vs. the I/R group. I/R, ischemia/reperfusion; IL-6, interleukin-6; IL-23, interleukin-23; TNFa, tumor necrosis factor-a; MDA, malondialdehyde; SOD, superoxide dismutase; ZQAE, aqueous extract of Citrus wilsonii Tanaka fruit . 
A
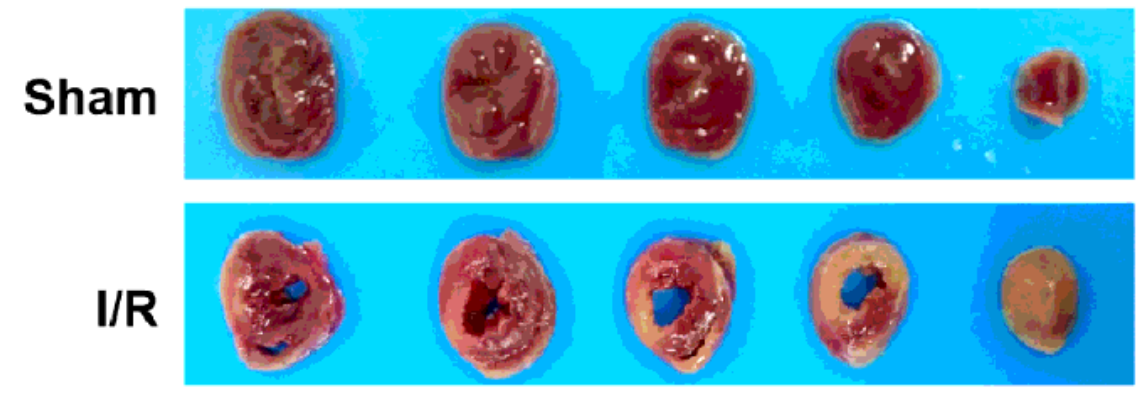

Naringin-I/R

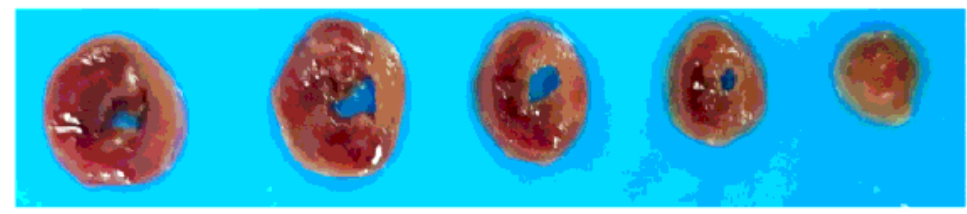

Ditiazem-I/R

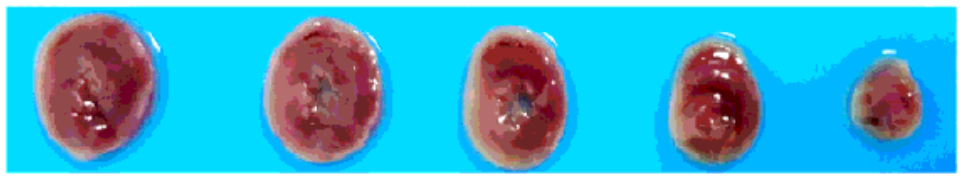

B

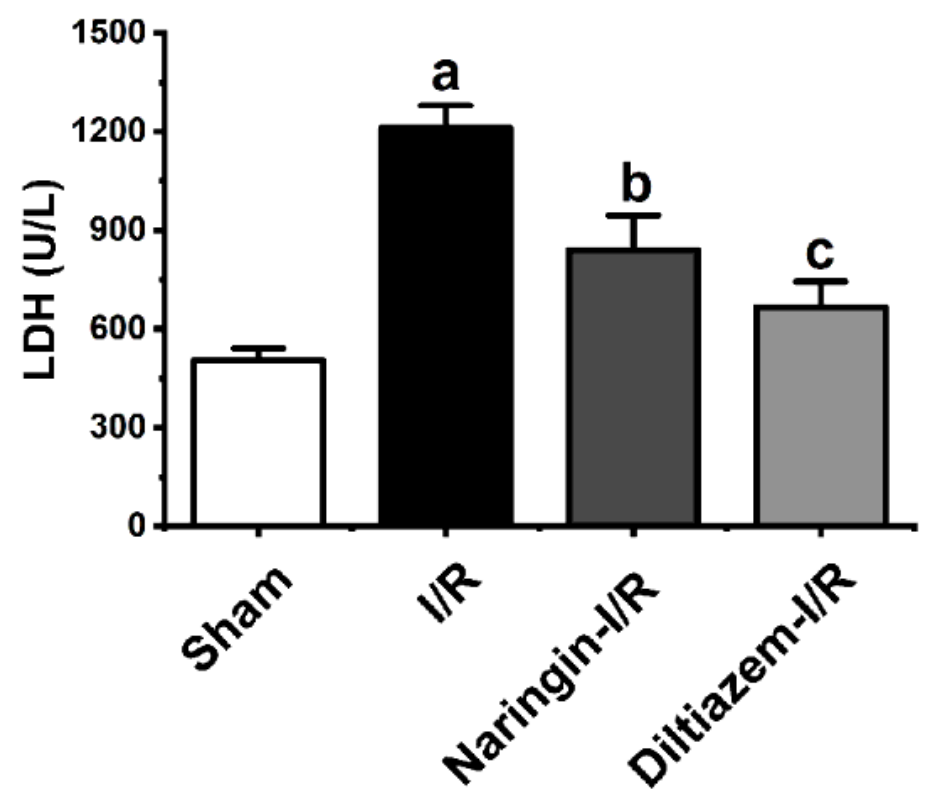

C

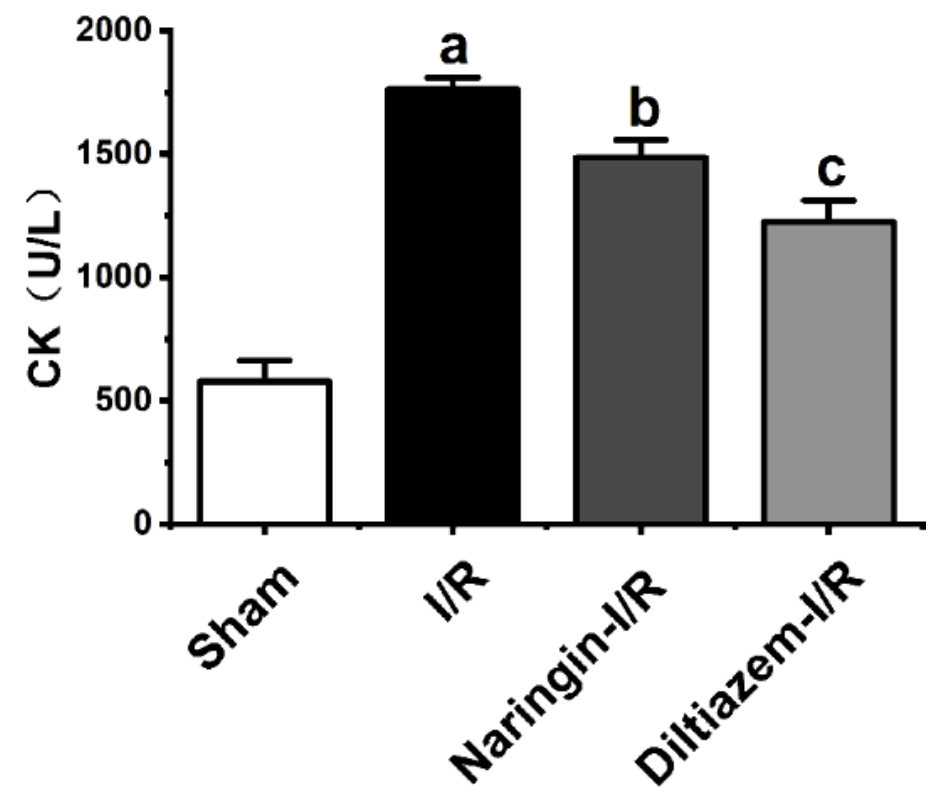

Figure 4

Naringin pretreatment reduced myocardial infarct size induced and improved ischemia/reperfusion injury in rats. (A) Naringin pretreatment reduced myocardial infarct size induced by $1 / R$ in rats, (B) Serum level of CK, (C) Serum level of LDH . a $P<0.05$ vs. the Sham group; b $P<0.05$ vs. the I/R group; $c P<0.05$ vs. the Naringin-l/R group (dose $5 \mathrm{mg} / \mathrm{kg}$ ). CK, Creatine kinase; LDH, Lactate dehydrogenase; I/R, ischemia/reperfusion; Sham, Sham-operated control. 
A

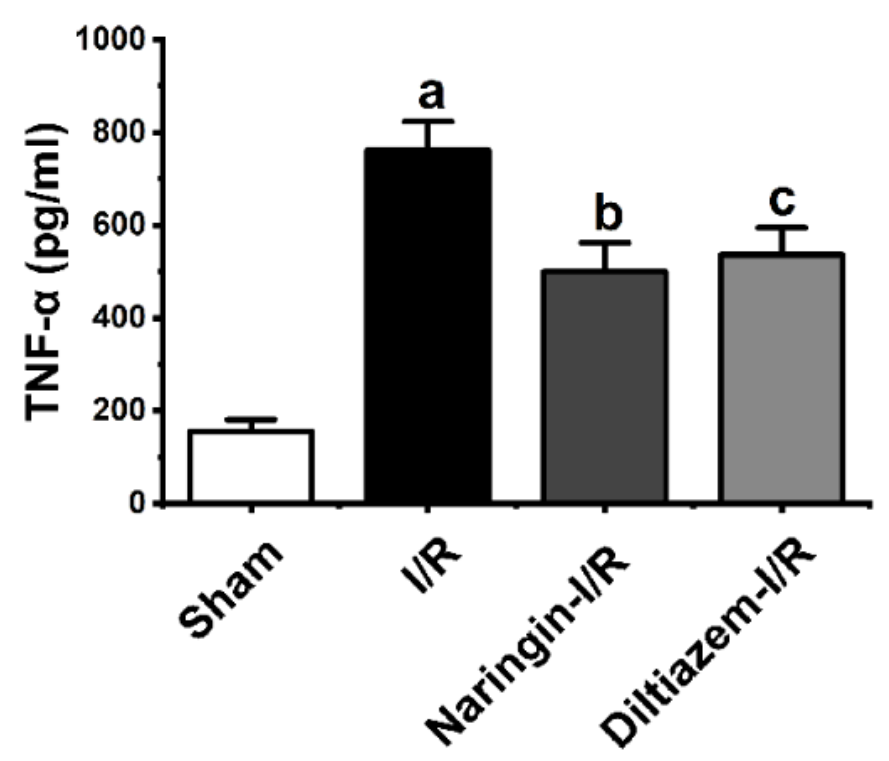

C

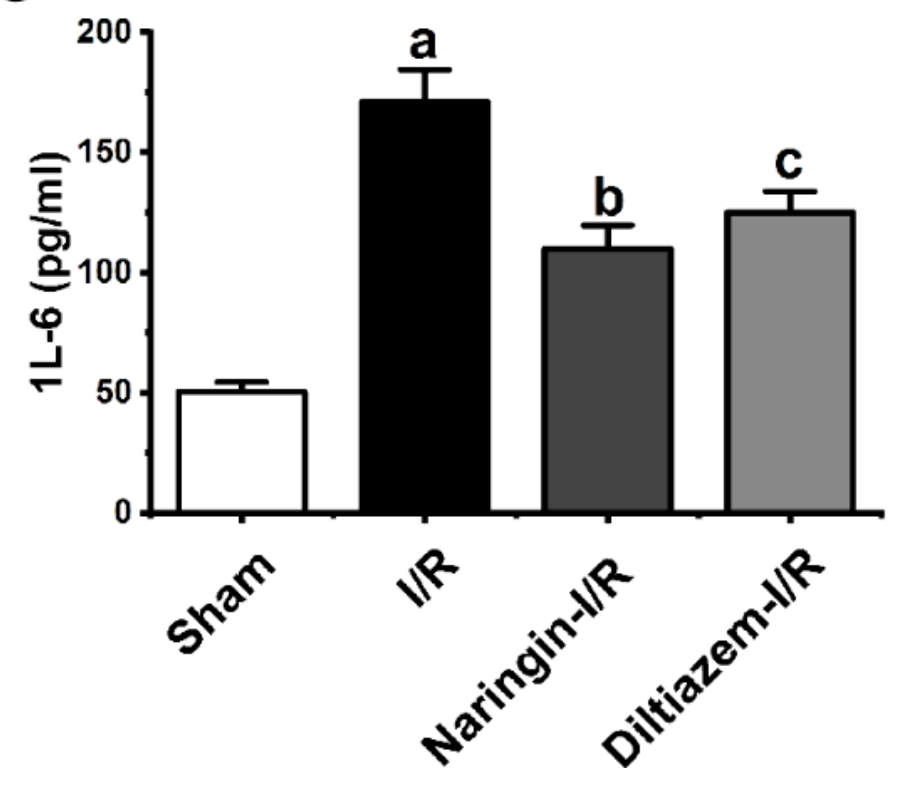

B

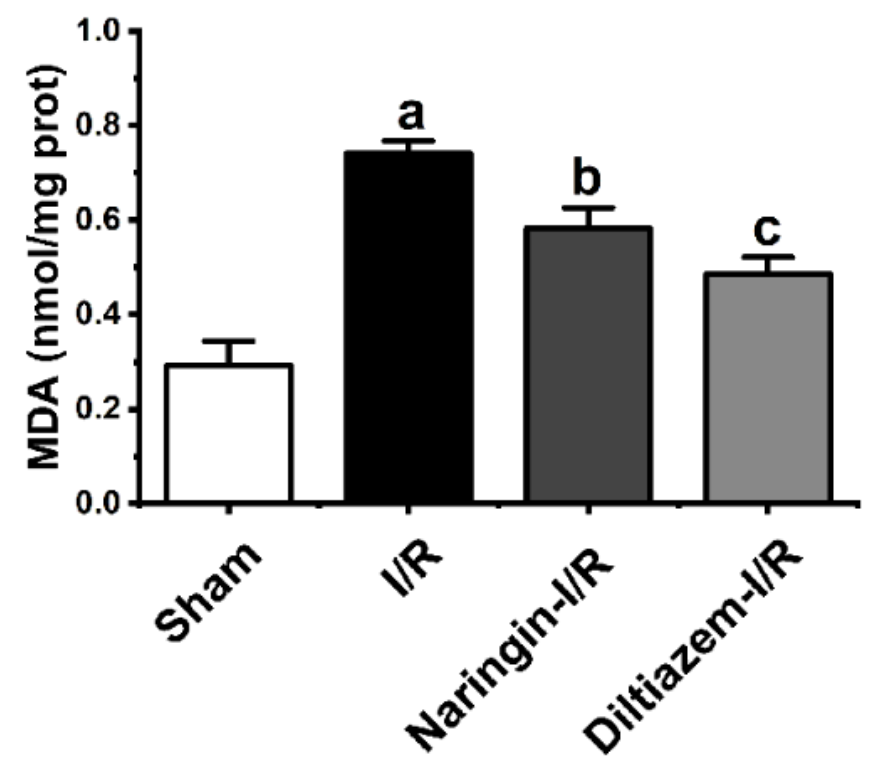

D

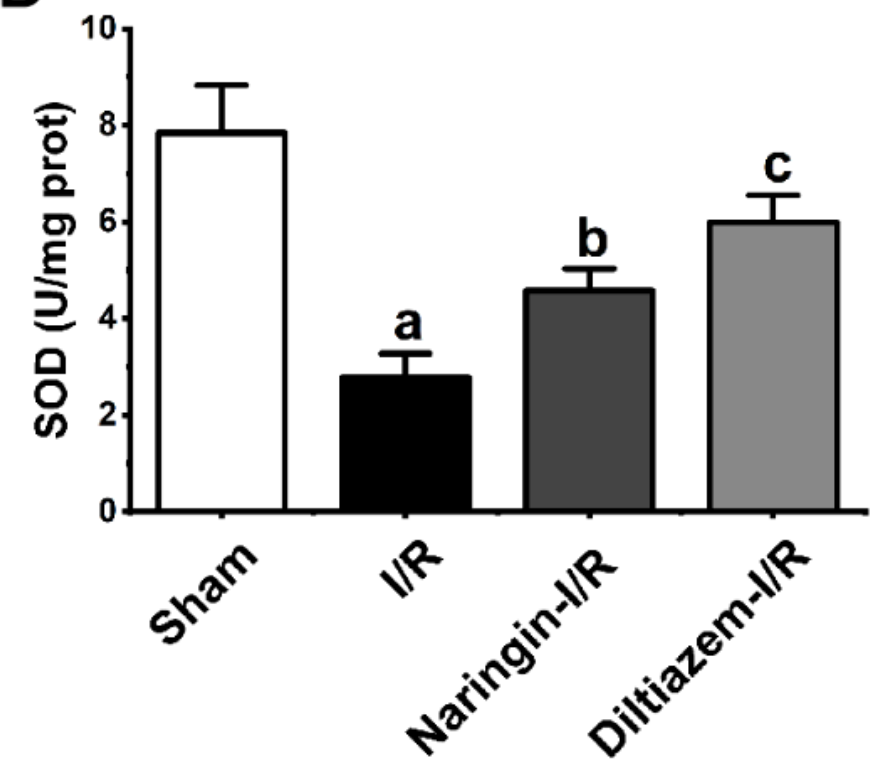

Figure 5

Naringin pretreatment can inhibit and reduce the level of inflammation and oxidative stress induced by I/R injury in rat myocardial. (A) Level of TNF-a, (B) MDA content, (C) Level of IL-6, (D) SOD activity. a $P<0.05$ vs. the Sham group; $b \quad P<0.05$ vs. the I/R group; $c P<0.05$ vs. the Naringin-I/R group (dose $5 \mathrm{mg} / \mathrm{kg}$ ). TNF-a, tumor necrosis factor-a; IL-6, interleukin-6; MDA, malondialdehyde; SOD, superoxide dismutase; l/R, ischemia/reperfusion; Sham, Sham-operated control. 
A

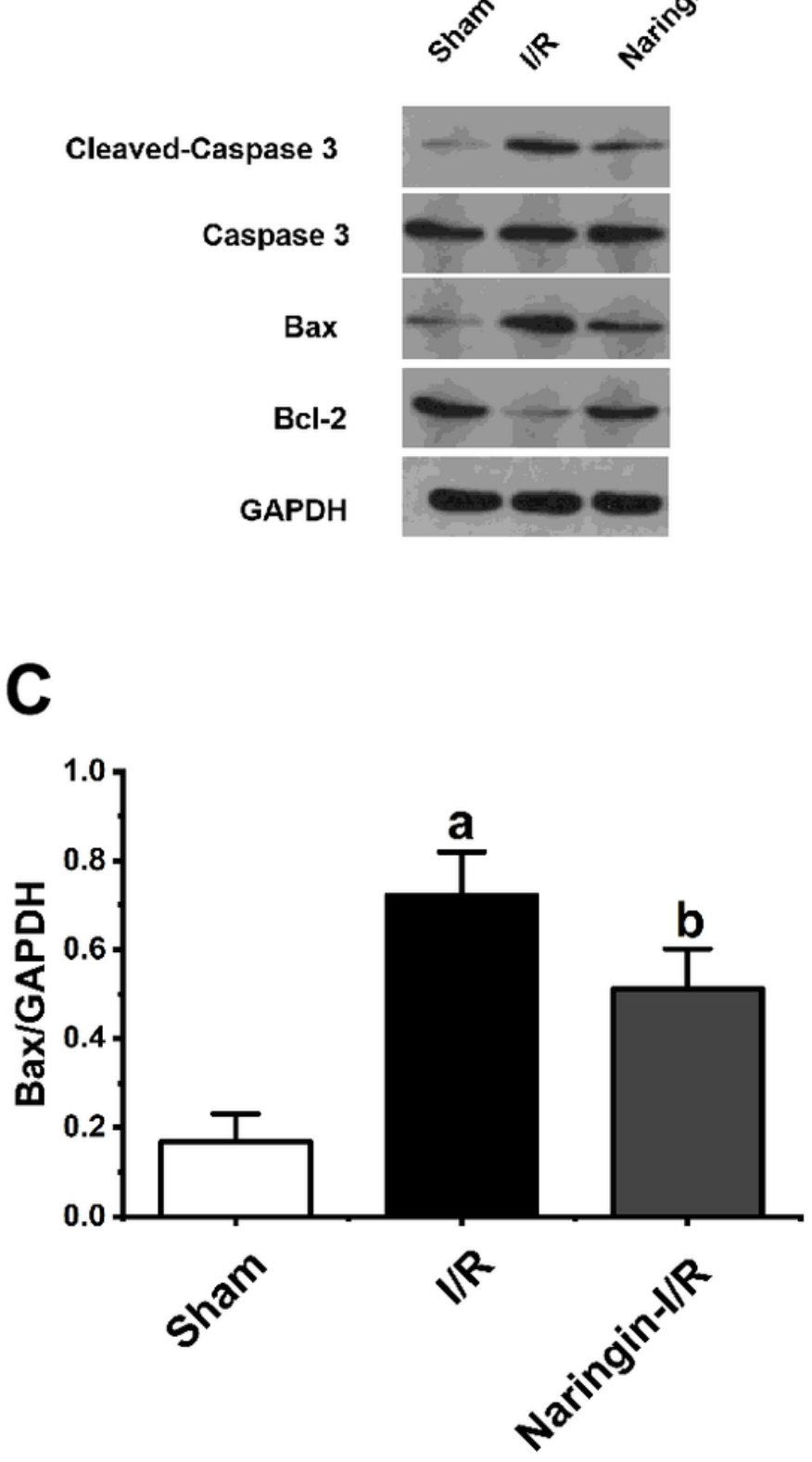

B
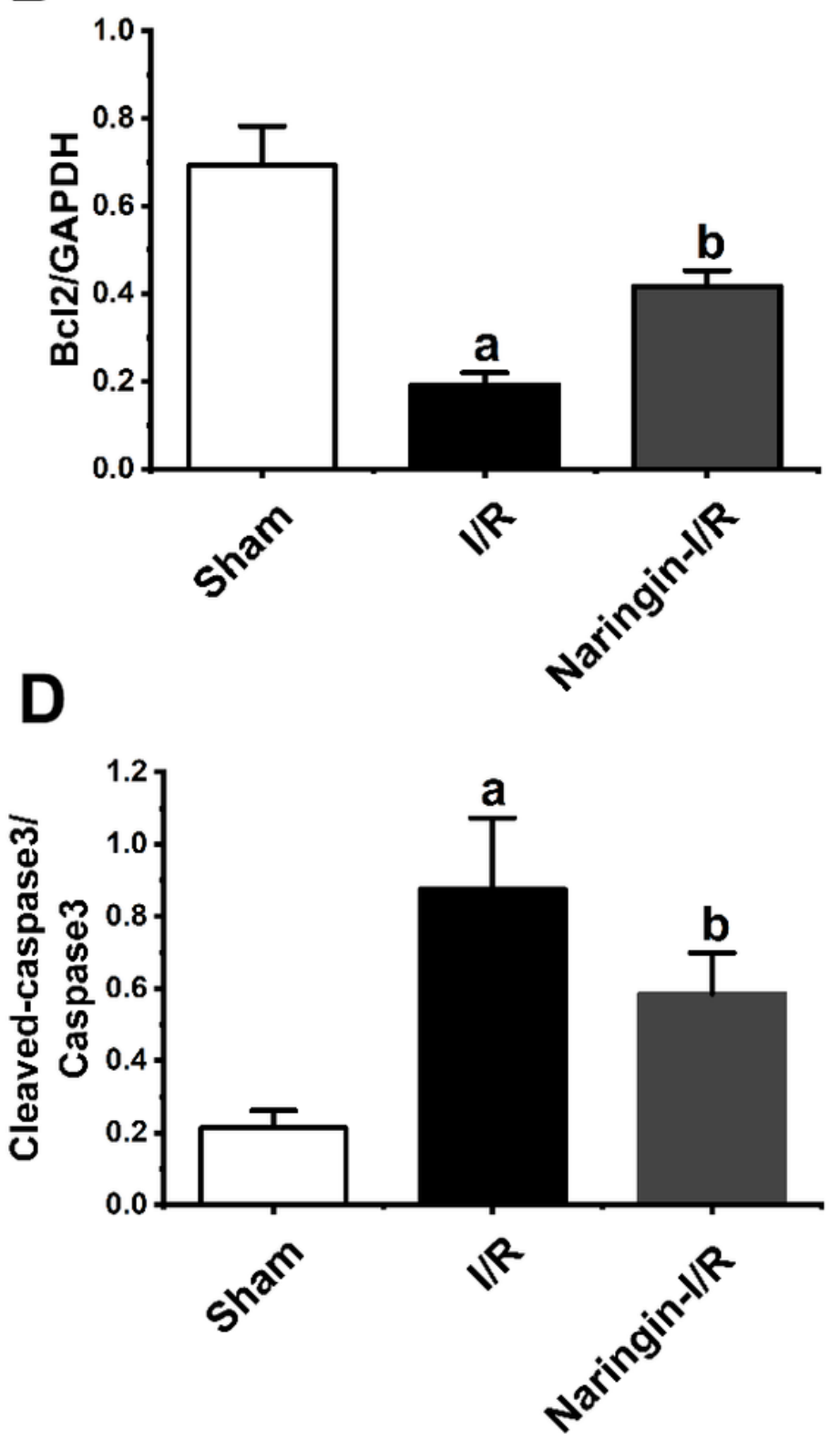

Figure 6

Naringin pretreatment inhibited the expression levels of myocardial apoptosis protein. (A) immunoblot results photo, (B) Bcl2 expression level, (C) Bax expression level, and (D) the ratio of cleaved Caspase 3/ total Caspaes 3. a $P<0.05$ vs. the Sham group; $b \mathrm{P}<0.05$ vs. the I/R group. I/R, ischemia/reperfusion; Sham, Sham-operated control. 

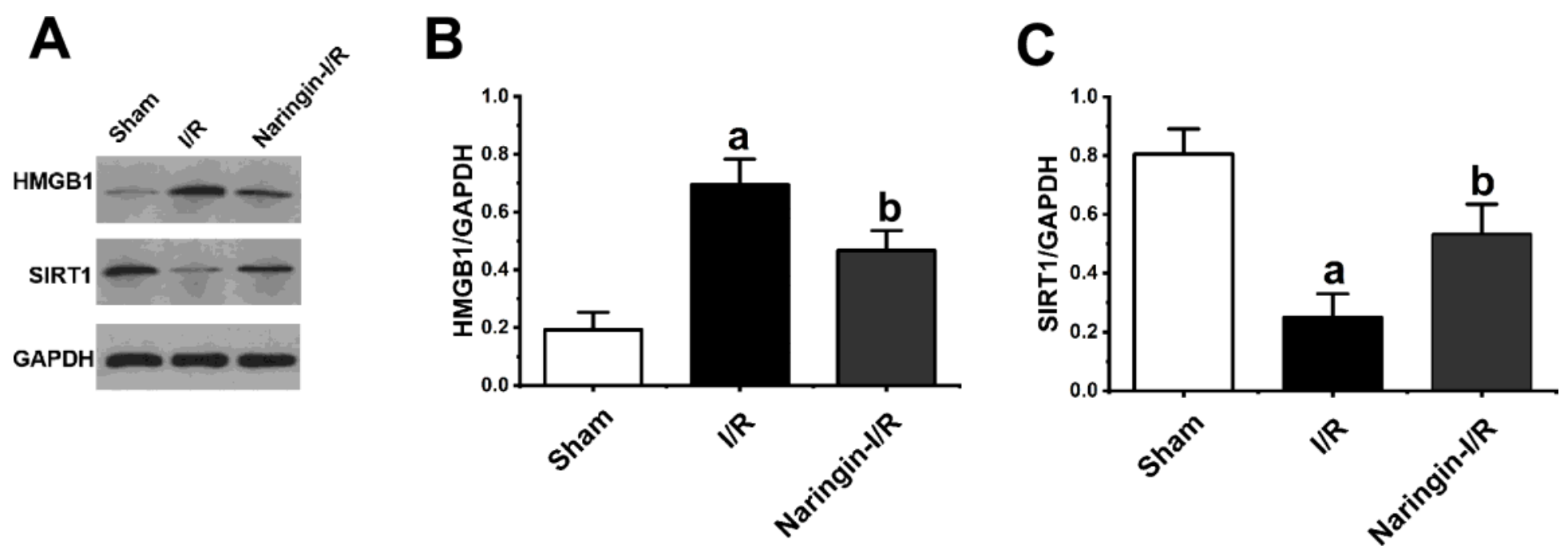

Figure 7

Naringin pretreatment inhibits the expression changes of HMGB1, SIRT1 in rat myocardium induced by I/R. (A) Images of western blot results, (B) Expression level of HMGB1, (C) Expression level of SIRT1. a $P<0.05$ vs. the Sham group; $b \quad P<0.05$ vs. the I/R group. I/R, ischemia/reperfusion; Sham, Sham-operated control.

\section{Supplementary Files}

This is a list of supplementary files associated with this preprint. Click to download.

- Supplementaloriginalsourcedata.pdf 